\title{
Irlande. Ambition et défis du nouveau plan pour l'éducation
}

Cathal de Paor

\section{(2) OpenEdition}

Journals

Édition électronique

URL : https://journals.openedition.org/ries/5580

DOI : 10.4000/ries.5580

ISSN : 2261-4265

Éditeur

France Education international

Édition imprimée

Date de publication : 1 décembre 2016

Pagination : 20-23

ISBN : 978-2-85420-612-8

ISSN : $1254-4590$

\section{Référence électronique}

Cathal de Paor, «Irlande. Ambition et défis du nouveau plan pour l'éducation », Revue internationale d'éducation de Sèvres [En ligne], 73 | décembre 2016, mis en ligne le 01 décembre 2018, consulté le 02 juillet 2021. URL : http://journals.openedition.org/ries/5580; DOI : https://doi.org/10.4000/ries.5580

Ce document a été généré automatiquement le 2 juillet 2021.

(c) Tous droits réservés 


\title{
le point sur...
}

\section{Irlande. Ambition et défis du nouveau plan pour l'éducation}

\author{
Cathal de Paor
}

1 L'année 2016 aura été une année charnière en Irlande, en matière d'éducation. Elle a été marquée, entre autres, par le lancement, en septembre, d'un plan d'action pour l'éducation, offrant un cadre général de développement. Mais les défis sont nombreux. L'un des chantiers qui ont suscité les plus fortes polémiques, ces derniers mois et années, est la réforme du secondaire inférieur. Si la réforme vise une meilleure prise en compte du contexte dans l'enseignement, cette démarche de contextualisation a suscité une forte opposition d'une partie du corps enseignant.

\section{Le plan d'action}

2 Assurer une éducation d'une excellente qualité pour tous : tel est l'objectif de l'Action Plan for Education 2016-2019 (DES, 2016), lancé en septembre 2016 par le nouveau ministre de l'éducation, Richard Bruton, nommé en mai, après l'installation d'un nouveau gouvernement suite à des élections. L'objectif du plan est d'améliorer le système, à travers une pluralité de stratégies et de programmes concernant tous les secteurs: petite enfance, scolarité obligatoire, enseignement et formation professionnels ainsi qu'enseignement supérieur.

3 Ces objectifs représentent ce que le ministère va entreprendre au cours des trois prochaines années, même si plusieurs actions sont déjà en cours, ayant été lancées par le ministre précédent. En gros, l'accent est mis sur les aspects suivants : briser les barrières pour les groupes présentant un risque d'exclusion; offrir une expérience d'apprentissage répondant aux standards internationaux les plus élevés; équiper les apprenants de tous âges, quelles que soient leurs capacités, de sorte qu'ils réussissent dans un monde en mutation; permettre à l'Irlande d'être un leader dans un large éventail de domaines scientifiques. Il s'agit d'assurer une éducation d'une excellente 
qualité pour tous, qui permette à chacun de s'épanouir pleinement et de contribuer utilement à la société.

4 Ancien ministre des emplois, de l'entreprise et de l'innovation, Richard Bruton propose un plan pour l'éducation qui comporte certaines caractéristiques d'un plan professionnel dans le monde de l'entreprise. Chaque action est associée à des indicateurs chiffrés permettant d'en mesurer l'efficacité. La thématique «entrepreneuriat, créativité et innovation» est identifiée comme un enjeu majeur, comme c'est le cas au sein d'organisations internationales telles que l'OCDE ou la Commission européenne. Certains ont pointé l'aspect managérial du plan, craignant une confusion entre son ancien et son nouveau portfolio par le ministre. Mais il faut noter que le plan met en avant le principe de la priorité accordée aux plus démunis et aux plus vulnérables.

5 Cette insistance sur le lien entre l'éducation est le monde professionnel est surtout mise en évidence dans les actions prévues concernant le développement de l'enseignement et de la formation professionnels (EFP). Ce secteur est actuellement prioritaire en Irlande, et a déjà fait l'objet de plusieurs réformes récentes. On vise une approche plus intégrée de l'offre, jusque-là fragmentée, et un lien plus étroit entre le monde de la formation et le monde du travail.

6 Une importante réforme du système, visant à assurer une meilleure adéquation entre offre de formation et besoins du marché du travail était intervenue en 2013, avec une restructuration des organismes de soutien. Le bénéfice de l'allocation des indemnités de chômage et la participation aux programmes d'activation du marché du travail sont désormais réunis, avec le transfert au ministère de la protection sociale de certains services de formation pour l'emploi, pour donner lieu à la création d'un seul service national. Celui-ci propose une aide et des conseils en matière de recherche d'emploi et de formation et de formation. Les indemnités peuvent être retirées si les demandeurs d'emploi refusent sans raison justifiée une offre d'emploi ou de formation. L'intégration des systèmes informatiques jusque-là séparés se poursuit également. Un système de profilage des chômeurs et l'organisation conjointe de sessions de groupe favorise une utilisation mieux ciblée des ressources, par une intervention précoce auprès des nouveaux bénéficiaires de prestations qui présentent un risque plus élevé de subir un chômage de longue durée. L'intégration des services offre l'occasion d'évoluer vers une plus grande qualité et pertinence dans l'offre de formation, puisque le département de protection sociale orienterait les demandeurs d'emploi, le cas échéant, vers les plus adaptées, qui pourraient être dispensées par des prestataires publics ou privés. Le nouveau service a pour mission de travailler en étroite collaboration avec la nouvelle autorité de l'éducation et de la formation professionnelle, SOLAS, ainsi qu'avec les comités régionales (Education and Training Boards) de façon à contribuer à une meilleure intégration de la protection sociale et de la formation.

\section{Les défis}

7 Mais les défis pour la réussite du plan sont nombreux. Dans l'enseignement supérieur, par exemple, les difficultés portent sur le financement, l'amélioration et la préservation de la qualité de l'enseignement, l'accès et la participation des élèves défavorisés ainsi que le nombre croissant d'étudiants. Le gouvernement poursuit également l'intégration 
de plusieurs instituts de technologie avec la création de nouvelles universités technologiques.

Le secteur de la petite enfance est également en pleine mutation, et doit répondre à la demande croissante de places, suite à la décision du gouvernement d'introduire la gratuité dans les crèches et les autres centres à partir de septembre 2016 pour chaque enfant dès l'âge de trois ans. Ces décisions ont des conséquences sur le recrutement du personnel, car un diplôme de formation de niveau 4 (cadre européen des certifications) sera nécessaire à partir de décembre 2016.

\section{La réforme du secondaire premier cycle}

Mais le chantier qui a déjà suscité les plus fortes polémiques, ces derniers mois, est la réforme du secondaire premier cycle. La réforme, désormais acceptée par l'un des deux syndicats concernés, est actuellement en cours dans les établissements où les membres de ce syndicat sont en exercice. Elle est censée favoriser une meilleure articulation entre le curriculum prescrit et le curriculum tel que mis en place par chaque établissement, et vise une meilleure prise en compte du contexte dans l'enseignement. Cette démarche de contextualisation suscite une forte opposition, en particulier en ce qui concerne l'évaluation des apprentissages.

10 En Irlande, les enseignements du secondaire (inférieur et supérieur) sont, en général, dispensés dans le même établissement. Le secondaire inférieur, le Junior Cycle, dure trois ans, et le Senior Cycle deux ou trois ans, selon le choix de l'élève. Le secondaire inférieur est sanctionné par un certificat de fin d'études, le Junior Certificate. Les sujets des épreuves sont communs à l'ensemble des établissements du territoire et sont notés dans un processus surveillé par la Commission d'État pour les examens (State Examinations Commission), une autorité publique placée sous l'égide du ministère de l'éducation. Pour la plupart des matières, les examens se déroulent au cours d'une seule séance, de généralement deux à trois heures, à l'exception des quatre matières suivantes : gaélique, anglais, mathématiques et études commerciales, pour lesquelles il y a deux examens. Le système d'évaluation est donc caractérisé par une dominance de l'évaluation terminale, avec une série d'examens d'une durée considérable.

11 Depuis la sortie d'un rapport (2011) du National Council for Curriculum and Assessment (NCCA) et d'un rapport ministériel, A framework for the junior cycle (DES, 2012), et suite à plusieurs périodes de négociations, la réforme a connu de nombreuses évolutions ce dernières années. Ce qui sous-tend la réforme est un questionnement sur le rôle du Junior Certificate, qui ne joue aucun rôle dans l'orientation des élèves dans le secondaire supérieur, et que beaucoup dépeignent comme une préparation en miniature de l'évaluation de fin d'études (que la grande majorité des élèves subira deux ou trois années plus tard). La première matière à avoir été introduite dans le cadre du nouveau cycle était l'anglais, en septembre 2014. En 2019, l'ensemble des 21 matières ou sujets auront été introduits.

12 La réforme vise donc à donner un plus grand rôle aux établissements dans l'élaboration de leur projet éducatif, en mettant l'accent sur le développement de huit nouvelles compétences des élèves, qui doivent leur permettre de réussir leur scolarité, leur orientation, ainsi que de s'adapter aux évolutions de leur vie personnelle, sociale et professionnelle. L'ensemble des connaissances, compétences, valeurs et attitudes 
nécessaires pour réussir le cycle serait présenté sous la forme d'un socle commun. Ce cadre a été conçu comme une tentative pour donner plus d'autonomie aux établissements dans la planification des programmes, pour privilégier des apprentissages approfondis et pour développer des compétences transversales. Au lieu de penser en termes de "couverture » des disciplines, l'enseignant et les élèves sont invités à penser en termes de maîtrise de certaines compétences transférables d'une situation à une autre. Une attention plus grande est portée au curriculum en tant qu'expérience construite sur le terrain, en mettant les enseignants et les élèves au centre du processus de changement, afin de renoncer à l'uniformité pour mieux s'adapter à la diversité des besoins des élèves.

\section{L'opposition à la réforme}

13 C'est la question de l'évaluation qui a suscité l'opposition la plus vive de la part des deux syndicats: l'Association of Secondary Teachers of Ireland (ASTI) la Teachers Union of Ireland (TUI). La réforme proposait d'introduire des épreuves ponctuelles de contrôle, tout au long de la formation, notées par les professeurs eux-mêmes dans les établissements, comme c'est le cas dans d'autres pays. Cette évaluation continue compterait pour $40 \%$ de l'évaluation, $60 \%$ étant réservés à l'examen final. Après une longue période de négociation, et conformément à l'accord entre le ministère et les syndicats en mai 2015 , les professeurs devaient mettre en œuvre l'évaluation en classe - mais, élément crucial, les résultats du contrôle continu ne figuraient pas dans le certificat de fin de cycle. Après l'avoir soumis au vote en septembre 2015, les membres de l'ASTI ont rejeté l'accord tandis que leurs collègues de l'autre syndicat, le TUI, l'ont accepté.

14 Le syndicat a souligné surtout le risque de renforcer les différences et les inégalités entre les établissements et de mettre à mal l'égalité des élèves face à l'offre éducative si les établissements avaient plus de liberté pour élaborer l'enseignement et l'évaluation. Favoriser des différences curriculaires entre les établissements aurait des conséquences défavorables pour la garantie de la qualité des apprentissages des élèves. Certains établissements pourraient adopter un "nouveau» curriculum, tandis que d'autres conserveraient en grande partie un curriculum plus « traditionnel».

15 La posture des deux syndicats est aussi liée à des questions plus larges sur les conditions de travail des enseignants. Dans un contexte de reprise économique modérée dans le pays, les syndicats enseignants (comme beaucoup d'autres dans le service public) poursuivent des négociations sur une éventuelle augmentation des salaires, suite aux réductions et aux gels imposés durant les années d'austérité. Là encore, les syndicats enseignants choisissent de se lancer dans des initiatives isolées.

16 Sans aucun doute, l'opposition à la réforme souligne la nécessité de réfléchir à la conception du métier d'enseignant, qui est sous-jacente à la réaction des acteurs envers le changement. Si la réforme est censée favoriser l'importance du contexte, c'est aussi l'occasion de repenser l'identité professionnelle des enseignants et les compétences dont l'enseignant aura besoin.

17 Le défi, depuis plusieurs décennies, a été de rendre l'éducation accessible à tous. Aujourd'hui, la difficulté, à l'intérieur des établissements, c'est de rendre la réussite 
accessible à tous. C'est l'une des idées fondatrices du plan d'action. Mais la réforme du secondaire inférieur jette un doute sur l'éventuelle réussite du plan d'action lui-même.

Il est indéniable que l'opposition des enseignants constitue un obstacle, surtout à la réalisation de certaines actions. La non-participation de l'un des syndicats, pour le moment en tout cas, risque de créer deux catégories d'établissements : ceux où les nouveaux programmes et modalités d'évaluation seront mis en place, et les autres établissements, où les anciens programmes continueront. Le système irlandais du secondaire inférieur se trouve donc dans une situation inédite car l'évaluation des élèves, dans un tiers des écoles environ, se fera comme auparavant. À l'heure où plusieurs pays optent pour une plus grande responsabilisation des établissements dans l'élaboration de leur curriculum, il reste à voir comment les partenaires éducatifs pourront parvenir à s'impliquer.

Mais la réussite du plan dépend avant tout de l'assainissement, en cours, des finances publiques. Le plan précise, à plusieurs reprises, que certaines actions seront mises en place «en fonction des ressources disponibles ». Tout dépend donc de la viabilité des finances publiques, encore déficitaires, mais en cours de redressement. En dépit de ces contraintes, le ministre a décidément fait un pas dans la bonne direction - même si la route est encore longue.

\section{BIBLIOGRAPHIE}

DES (2016) : Action plan for education, Dublin : Department of Education and Skills. [en ligne] [https://goo.gl/gzNXpx]

DES (2012) : A Framework for Junior Cycle, Dublin : Department of Education and Skills. [en ligne] [https://goo.gl/1X81eu]

NCCA (2011) : Towards a framework for junior cycle, Dublin : National Council for Curriculum and Assessment (NCCA). [en ligne] [https://goo.gl/817AC]

\section{INDEX}

Mots-clés : enseignement secondaire, réforme de l'enseignement

Index géographique : Irlande

Keywords : secondary education, educational reform

Palabras claves : enseñanza secundaria, reforma de la educación

\section{AUTEUR}

\section{CATHAL DE PAOR}

Cathal de Paor est directeur de la formation continue des enseignants au Mary Immaculate College, Université de Limerick, Irlande. Titulaire d'un doctorat en sciences de l'éducation de 
l'Université de Nantes, il a participé à de nombreux comités d'évaluation, en tant qu'évaluateur des cursus de formation dans des établissements d'enseignement supérieur en Irlande et en Europe. Ancien professeur des écoles primaires, il a travaillé sur l'élaboration des programmes scolaires auprès du conseil national pour les programmes en Irlande. Courriel:

Cathal.dePaor@mic.ul.ie 